\title{
Enzymological and physiological consequences of restructuring the lipoyl domain content of the pyruvate dehydrogenase complex of Escherichia coli
}

\author{
John R. Guest, Margaret M. Attwood, Rosane S. Machado, \\ Khalil Y. Matqi, John E. Shaw and Sarah L. Turner
}

Author for correspondence: John R. Guest. Tel: +44 114282 4406/3. Fax: +44 1142728697.

The Krebs Institute for Biomolecular Research, Department of Molecular Biology and Biotechnology, University of Sheffield, Sheffield S10 2TN, UK

\begin{abstract}
The core-forming lipoate acetyltransferase (E2p) subunits of the pyruvate dehydrogenase (PDH) complex of Escherichia coli contain three tandemly repeated lipoyl domains although one lipoyl domain is apparently sufficient for full catalytic activity in vitro. Plasmids containing IPTG-inducible aceEF-IpdA operons which express multilip-PDH complexes bearing one $\mathrm{N}$ terminal lipoyl domain and up to seven unlipoylated (mutant) domains per E2p chain, were constructed. Each plasmid restored the nutritional lesion of a strain lacking the PDH complex and expressed a sedimentable PDH complex, although the catalytic activities declined significantly as the number of unlipoylated domains increased above four per E2p chain. It was concluded that the extra domains protrude from the 24-meric E2p core without affecting assembly of the E1p and E3 subunits, and that the lipoyl cofactor bound to the outermost domain can participate successfully at each of the three types of active site in the assembled complex. Physiological studies with two series of isogenic strains expressing multilip-PDH complexes from modified chromosomal pdh operons (pdhR-aceEF-IpdA) showed that three lipoyl domains per E2p chain is optimal and that only the outermost domain need be lipoylated for optimal activity. It is concluded that the reason for retaining three lipoyl domains is to extend the reach of the outermost lipoyl cofactor rather than to provide extra cofactors for catalysis.
\end{abstract}

Keywords: pyruvate dehydrogenase complex, lipoate acetyltransferase, lipoyl domains, protein engineering, metabolic engineering

\section{INTRODUCTION}

Escherichia coli uses three enzymes for catalysing pyruvate oxidation under different physiological conditions. The pyruvate dehydrogenase (PDH) complex operates during aerobic respiratory growth but its function is increasingly adopted by pyruvate formatelyase (PFL) under anaerobic conditions. Thus, the PDH complex and PFL are both functional during anaerobic respiratory growth with nitrate (Kaiser \& Sawers, 1994), whereas PFL takes over completely during fermentative growth. In contrast, pyruvate oxidase (PoxB), which

Abbreviations: $D$, dilution rate; E1p, pyruvate dehydrogenase, E2p, lipoate acetyltransferase; E3, lipoamide dehydrogenase; PDH, pyruvate dehydrogenase (complex). converts pyruvate directly to acetate rather than to acetyl-CoA, is an aerobic stationary-phase enzyme (Chang et al., 1994). These enzymes control the flux of glycolytic carbon into growth-related products, including the products of heterologous gene expression, as well as normal metabolites. The ability to manipulate the synthesis or activity of these enzymes offers the possibility of redirecting substrate carbon into biotechnologically useful products. It could also provide a means of redirecting substrate carbon away from acetate, which accumulates at high cell density and by uncoupling membrane potential, is a major cause of poor productivity and cell death (Cutayer \& Poillon, 1989; Konstantinov et al., 1990). One strategy for manipulating pyruvate metabolism is to replace the pyruvate-inducible regulatory region of the $p d h$ operon ( $p d h R-a c e E-a c e F-l p d A$; Fig. 1) by the IPTG-regulated 


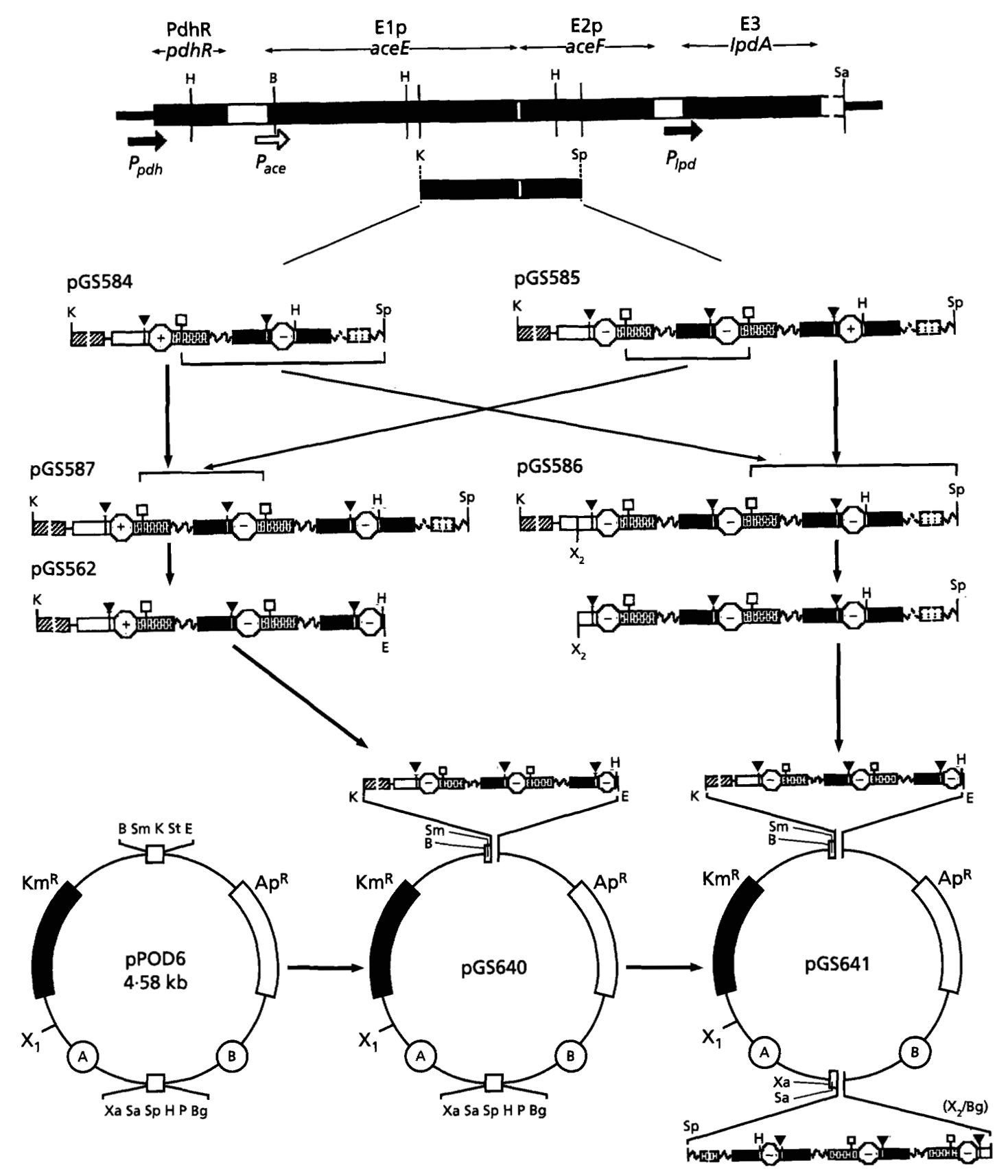

Fig. 1. Construction of a dual replicon (pGS641) containing two fragments of the aceF region encoding tandem wild-type and mutant lipoyl domains. Restriction map of the $p d h R$-aceEF-IpdA region (not to scale) showing the location of the $K p n I-5 p h l$ cassettes encoding lipoyl domains with wild-type $(+)$ and mutant $(-)$ lipoyl-binding sites (different origins are denoted by the shaded boxes: lip1, open; lip2, hatched; and lip3, filled) and the associated linkers (zig-zag lines). The Kpnl-EcoRI insert (+ + ) of pGS640 was derived by sub-cloning the $300 \mathrm{bp} \mathrm{Accl} \mathrm{fragment} \mathrm{of} \mathrm{pGS585} \mathrm{into} \mathrm{pGS584} \mathrm{in} \mathrm{the}$ desired orientation, and then sub-cloning the Kpnl-Hindlll fragment of pGS587 into pUBS1 to pick up a flanking EcoRI site for ligation into the dual replicon, PPOD6. The Xholl-Sphl insert (- - ) of pGS641 was constructed in pGS586 by

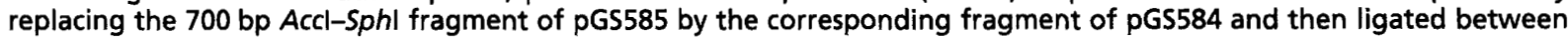
the Bg/ll and Sphl sites of pGS640, where the Bg/ll site was unexpectedly lost. Abbreviations/symbols: $\square, A c c l ; \nabla, B C l l ; B$, BamHl; Bg, Bglll; E, EcoRI; H, Hindlll; K, Kpnl; P, Pstl; Sa, Sall; Sm, Smal; Sp, Sphl; St, Sstl; X, Xhol; X Xholl.

tac promoter so as to control synthesis of the PDH complex by the concentration of added IPTG (Cassey et al., 1996; Guest et al., 1996). An alternative strategy is to replace the genes encoding catalytic subunits by genetically manipulated variants, thus to alter the efficiency of the PDH complex (Davé et al., 1995). The second approach has now been extended to include further modifications of the PDH complex and at the same time, to use physiological criteria for assessing structure-function relationships of the complex. 
The PDH complex of E. coli catalyses the NAD ${ }^{+}$-linked oxidative decarboxylation of pyruvate to acetyl-CoA by three integrated reactions (Guest et al., 1989; Perham, 1991; Mattevi et al., 1992). It contains three enzymic subunits encoded by the aceE, aceF and $l p d A$ genes of the $p d h$ operon: pyruvate dehydrogenase (E1p, EC 1.2.4.1), lipoate acetyltransferase (E2p, EC 2.3.1.12) and lipoamide dehydrogenase (E3, EC 1.8.1.4), respectively (Fig. 1). Multiple copies of the dimeric E1p and E3 components are assembled on the respective edges and faces of a cubic core containing 24 E2p subunits, the overall chain stoichiometry (E1p:E2p:E3) being approximately $1 \cdot 0: 1 \cdot 0: 0 \cdot 5$. The E2p subunits contain three similar lipoyl domains (lip) connected to each other and to a small E3-binding domain (E3bd), by conformationally mobile linkers (xxxx, where $\mathrm{x}$ denotes about five residues), and a C-terminal catalytic domain (CAT):

\section{$\mathrm{NH}_{2}$-lip1-xxxx-lip2-xxxx-lip3-xxxxx- E3bd-xxx-CAT-COOH}

The CATs aggregate to form a 24-meric inner core from which the lipoyl domains protrude. Each lipoyl domain contains a covalently bound lipoyl cofactor that is attached post-translationally to a prominent lysine residue either by lipoate protein ligase which uses preformed lipoate and ATP, or by an alternative mechanism which takes lipoyl groups directly from the de novo biosynthetic pathway (Green et al., 1995; Morris et al., 1995). The lipoyl cofactors project from the inner core and approach the acetyltransferase active sites from outside the core whereas CoA enters the catalytic channel from within the core (Mattevi et al., 1992). All of the lipoyl cofactors are functional and the mobility inherent in the 'domain-linker' organization and the lipoyllysine 'swinging arm' is presumed to facilitate their reductive acetylation, deacetylation and re-oxidation at the E1p, E2p and E3 catalytic sites in the assembled complex.

The numbers of lipoyl domains per E2p chain varies from one to three in different 2-oxo acid dehydrogenase complexes and it is not clear why three are retained by the PDH complexes of E. coli and other Gram-negative bacteria, e.g. Azotobacter vinelandii (Schulze et al., 1992). Site-directed deletion of the lip segments encoding one or two lipoyl domains has no detectable effect on specific activity or active-site coupling, but the catalytic activity is abolished when all three lip segments are deleted or when the lipoyl domains are rendered unlipoylatable by lipoyl-Lys $\rightarrow$ Gln substitutions (Guest et al., 1985; Allen et al., 1989). No mandatory order of reductive acetylation is apparent because complexes containing mixtures of wild-type and mutant lipoyl domains $(+-;-+;++-)$ are fully active, although some impairment is associated with the --+ arrangement (Allen et al., 1989). Activity is also impaired (but not abolished) by increasing the lipoyl domain content to between four to nine per E2p chain, possibly due to under-lipoylation of the domains participating in catalysis and to steric interference from unlipoylated domains (Machado et al., 1992). An important feature contributing to a high catalytic efficiency is the conformational mobility conferred by the linker sequences. This is apparent from the impairment that accompanies the shortening or replacing of the residual lipoyl linker of a 1lip-PDH complex, and the enhancement afforded by incorporating a polyproline linker (Miles et al., 1988; Turner et al., 1993). High-field $\mathrm{H}^{1}$-NMR studies with variants containing from zero to nine lipoyl domains per $E 2 p$ chain gave the first indication that the mobility of the lipoyl linkers relative to the domains, and hence the catalytic efficiency, is highest in the wild-type complex (Machado et al., 1993). The superiority of the 3lip-PDH complex has since been confirmed in physiological studies with stable isogenic strains that express 1lip-, 2lip- and 3lip-PDH complexes, in which it was concluded that lowering the lipoyl domain content adversely affects growth rate and yield (Davé et al., 1995). In the present report, the physiological consequences of increasing the number of lipoyl domains from three to nine per E2p chain, and the effects of inserting up to seven unlipoylatable (mutant) domains between a wildtype N-terminal lipoyl domain and the E3-binding domain, were investigated. The results show the extent to which a single lipoyl cofactor can be distanced from the cognate active sites yet retain activity, and they help to explain why the wild-type E2p chain retains three lipoyl domains.

\section{METHODS}

Bacteria, plasmids and cloning procedures. The strains of E. coli K-12 were: W3110 (iclR trpR), the prototrophic strain; JRG2390 (aceF:: $k a n^{\mathrm{R}}$ ), a derivative of W3110 in which the aceF gene was inactivated by a net deletion of two lip segments and insertion of $\mathrm{a} \mathrm{kan}^{\mathrm{R}}$ cassette into the residual lip segment (Davé et al., 1995); JRG2931-2933 (1lip-3lip), derivatives of a spontaneous $\mathrm{Nal}^{\mathrm{R}}$ mutant of JRG2930 in which the aceF:: $k a n^{\mathbf{R}}$ region was replaced by aceF genes encoding E2p subunits with one to three lipoyl domains (Davé et al., 1995); JRG3134-3139 (4lip-9lip) and JRG3162-3168 (2lip-8lip), analogous derivatives of JRG2930 containing aceF genes with multiple lip segments (this work); DH5 $\alpha$ (AlacU169 $\phi 80$ lac $Z \Delta \mathrm{M} 15$ bsdR17 endA1 recA1), the routine transformation host (Sambrook et al., 1989); JC7623 (recB21 recC22 $s b c B 15 s b c C 201$ ), for chromosomal gene replacement (Oden et al., 1990); GM242 (dam-3 recA1), for producing plasmids sensitive to $B c l$ I digestion; and JRG1342 [ $\Delta($ aroP-pdhRaceEF-lpdA) 18 pox recA1], for over-producing modified $\mathrm{PDH}$ complexes and for nutritional tests (Guest et al., 1985).

The dual replicon pPOD6 containing replication origins from pACYC184 (A) and pIJ2925 (B) linked to $\mathrm{Km}^{\mathrm{R}}$ and $\mathrm{Ap}^{\mathrm{R}}$ determinants (respectively) and two different multi-cloning sites (Fig. 1), was kindly provided by A. F. C. Perry and N. J. Brown (University of Birmingham, unpublished).

The KpnI-SphI cassettes containing multiple lip segments (aceF sub-genes) were constructed from those of pGS231 $(+-)$ and pGS234 $(--+)$ (Allen et al., 1989) after transfer to the intermediary vector (pT7T3 18U, Pharmacia) to generate pGS584 $(+-)$ and pGS585 $(--+)$, respectively (Fig. 1). These plasmids were used as sources of the KpnIEcoRI (+ - ) and XhoII-SphI ( - - ) cassettes of pGS562 and pGS586 (respectively) which were transferred sequentially to pPOD6 to generate pGS641 via pGS640, as summarized in Fig. 1. Plasmid pGS641 was maintained in DH5 $\alpha$ with selection 

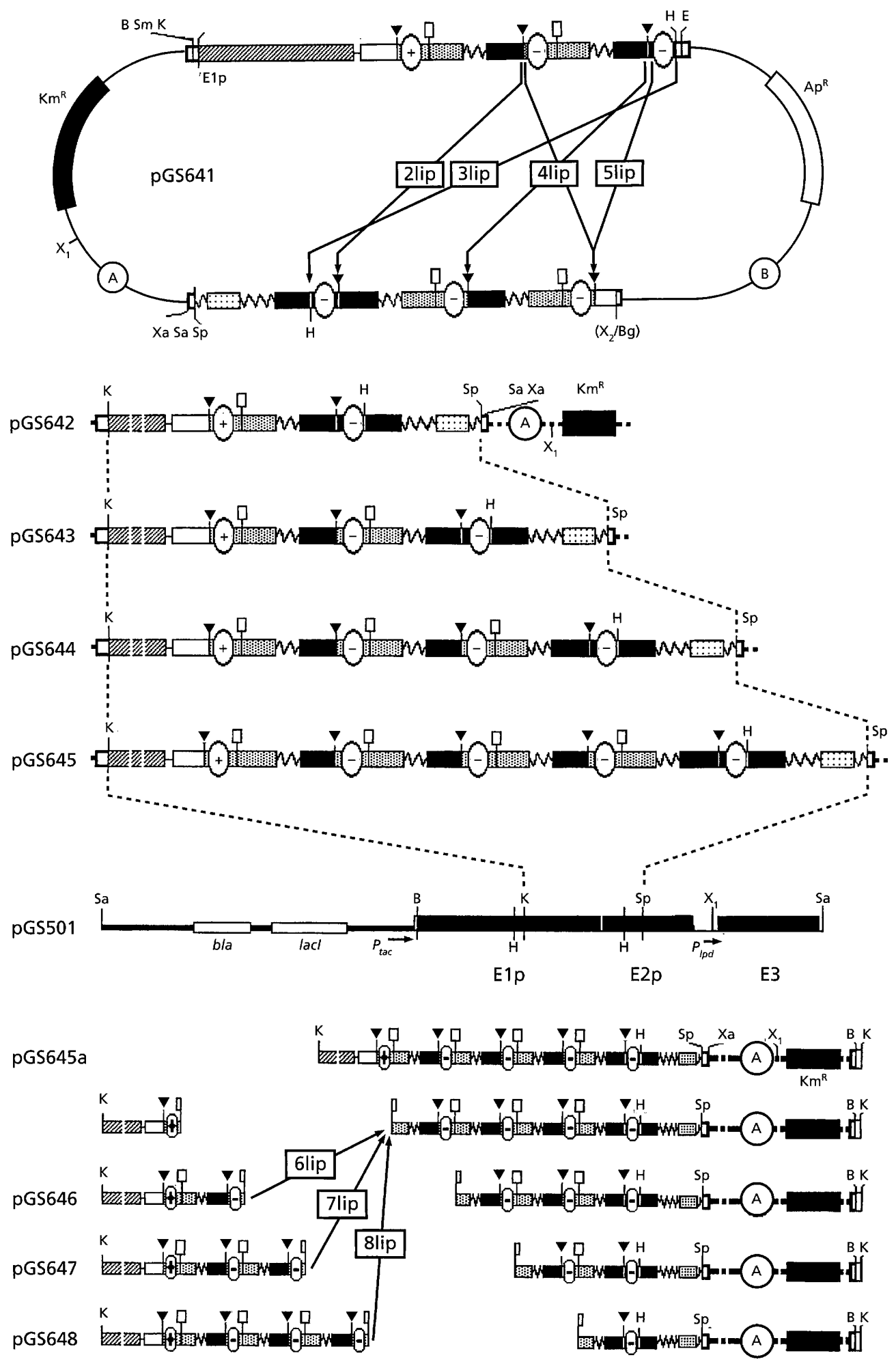

Fig. 2. Construction of expression plasmids encoding PDH complexes with two to eight lipoyl domains per E2p chain. Kanamycin-resistant resolvants of pGS641 were generated by partial digestion with $B c / l(\nabla)$, or complete digestion with HindIII (3lip only) and re-ligation to give pGS642-645. A derivative of pGS645 lacking the vector Sall/Accl site (pGS645a), was constructed by Sall cleavage, end-filling and re-ligation, linearized with $K p n l$, and partially digested with Accl ( $\square$ ) to yield fragments that can be combined to form plasmids encoding higher orders of lipoyl domains (pGS646-648), as indicated. The Kpnl-Sphl cassettes from each intermediary plasmid (pGS642-648) were transferred to pGS501, a $P_{\text {tas }}$ expression plasmid and cassette-replacement receptor for the aceEF-IpdA operon, to generate the corresponding set of expression plasmids pGS662-668. Abbreviations/symbols as in Fig. 1. 
for both antibiotic markers to prevent segregation of the two replicons by internal crossing-over between homologous regions of the sub-cloned segments. The expression plasmid, pGS501 (Russell et al., 1992), in which an aceE-ace $F_{n i p}-l p d A$ operon is expressed from the IPTG-inducible tac promoter (Fig. 2), served as receptor for the original and restructured $K p n \mathrm{I}-S p h \mathrm{I}$ cassettes of pGS642-648 to generate pGS662-668 (Fig. 2). Analogous expression plasmids containing operons with zero to nine wild-type lip segments were constructed previously (Machado et al., 1992): pGS489 (Olip), pGS367 (1lip), pGS524 (2lip), pGS523 (3lip), pGS504 (4lip), pGS505 (5lip) and pGS556-559 (6lip-9lip). Other plasmids were: pUBS1, a derivative of pUC19 containing the multi-cloning site from pBluescriptSK (Dr G. Murphy, Institute of Plant Science Research, Cambridge) used in sub-cloning; pUC4K+, a derivative of pUC4K (Pharmacia) in which the kan $^{\mathrm{R}}$ gene is flanked by two multi-cloning sites from pUC19, used as the source of the $\mathrm{kan}^{\mathrm{R}}$ cassette; and pMAK705 $\left(\mathrm{Ap}^{\mathrm{R}}, \mathrm{Cm}^{\mathrm{R}}\right)$, thermo-sensitive replicon used for chromosomal gene replacement (Hamilton et al., 1989). Standard molecular genetic procedures (Sambrook et al., 1989) were used, JRG2930 and JRG1342 were transformed by electroporation, and transduction with P1vir1 was used for strain construction (Miller, 1972).

Media, nutritional tests and enzymology. Cultures were grown in L broth plus glucose $(6 \mathrm{mM})$ supplemented appropriately with ampicillin $\left(50 \mu \mathrm{g} \mathrm{ml}^{-1}\right)$ or kanamycin $(20 \mu \mathrm{g}$ $\mathrm{ml}^{-1}$ ). Nutritional tests were performed with plates of glucose (11 $\mathrm{mM}$ ) minimal media supplemented with acetate $(2 \mathrm{mM})$, thiamin $\left(10 \mu \mathrm{g} \mathrm{ml}^{-1}\right)$, L-methionine $\left(10 \mu \mathrm{g} \mathrm{ml}^{-1}\right)$, ampicillin $\left(25 \mu \mathrm{g} \mathrm{m}^{-1}\right)$ and IPTG $(20 \mu \mathrm{M})$, as required (Guest et al., 1985). The over-production and purification of PDH complexes from transformants of JRG1342 involved induction with IPTG $(60 \mu \mathrm{M})$, ultrasonic disruption, sedimentation from crude extracts and FPLC ion-exchange chromatography (Mono-Q), as described by Russell et al. (1992). Specific activities [in units (mmol NADH formed) ${ }^{-1} \mathrm{mg}$ protein $)^{-1}$ ] of the PDH complexes, were calculated from the rates of pyruvate-dependent reduction of $\mathrm{NAD}^{+}$(Russell \& Guest, 1990); mean values for three independent preparations are quoted. $M_{\mathrm{r}}$ values and subunit stoichiometries were estimated by SDS-PAGE $(0 \cdot 1 \%$ SDS, $7.5 \%$ resolving gel, $4.5 \%$ stacking gel, with $3 \mu \mathrm{g}$ protein in each lane) and densitometric analysis (Bio-Profil) after staining with Coomassie Brilliant Blue R250. The procedure for lipoylating purified PDH complexes with lipoyl protein ligase has been described by Brookfield et al. (1991) and Green et al. (1995).

Chromosomal replacement procedures. The chromosomal aceF gene of $E$. coli W3110 was replaced by altered genes containing multiple wild-type lip segments using derivatives of thermo-sensitive replicon, pMAK705 (Hamilton et al., 1989). The procedure was similar to that used previously for lowering the number of lip segments (Davé et al., 1995) except that a new series of pMAK705-derivatives having a larger aceF-lpd flanking region was used in order to improve the frequency of chromosomal replacement. The relevant plasmids were constructed by first cloning the $4.6 \mathrm{~kb} K p n \mathrm{I}-$ SalI fragment of pGS367 (Russell et al., 1992) containing a wild-type aceEFlpdA segment into pUBS1 to provide the flanking HindIII site in pGS779, and then transferring the $2.32 \mathrm{~kb}$ SpbI-HindIII aceF-lpdA fragment to pMAK705 to generate pGS780 (Fig. 3). The $1.3 \mathrm{~kb} \mathrm{kan}{ }^{\mathrm{R}}$ cassette of pUC4K + was inserted at the vector $\mathrm{Bam} \mathrm{HI}$ site to provide a convenient phenotypic marker in the product, pGS781, which was readily replaced by $K p n I-S p h \mathrm{I}\left(a c e^{\prime} E F^{\prime}\right)$ segments from the 'multilip' expression plasmids (pGS504, -505, -556-559) at the same time

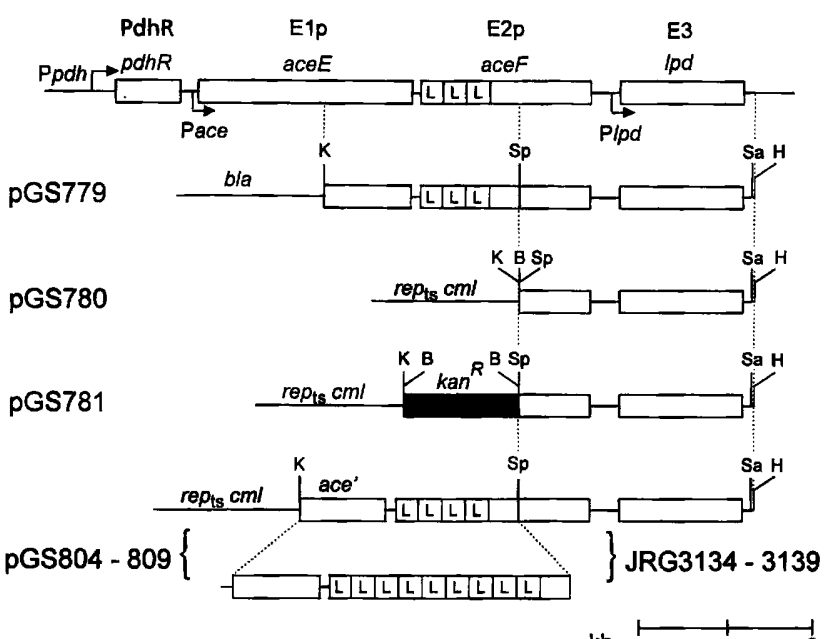

$\mathrm{kb} \stackrel{\longmapsto}{0}$

Fig. 3. Construction of isogenic strains containing single copies of genetically modified $p d h$ operons. The genes and promoters of the wild-type pdh operon in which the aceF gene includes three lip segments (L), are shown at the top. Derivatives of the pMAK705 $\left(r e p_{\mathrm{ts}} \mathrm{cml}\right)$ containing segments of the operon used in modifying the chromosomal acef gene (numbered on the left) and the corresponding strains (numbered on the right), were constructed and used as described in Methods. The kan $^{R}$ cassette is shaded and relevant restriction sites are: $\mathrm{B}, \mathrm{BamHI}$; H, HindIII; K, Kpnl; Sa, Sall; and Sp, Sphl.

restoring continuity to the aceEF-lpdA region (Fig. 3). This provided a series of appropriately modified thermo-sensitive replicons (pGS804-809) with which to generate the corresponding chromosomally replaced strains (JRG3134-3139, 4lip-9lip) by sequential co-integration into, and resolution from, the chromosome of JRG2930 (aceF::kan $\left.{ }^{\mathrm{R}} \mathrm{Nal}^{\mathrm{S}}\right)$, as described by Davé et al. (1995).

An analogous series of isogenic strains containing ace $F$ genes with one wild-type lip segment and up to seven mutant lip segments, was made by the method of Oden et al. (1990). A transformation host, JRG3141 ( $\operatorname{cecBC} s b c B C$ aceF::kan $\left.{ }^{\mathrm{R}}\right)$, was first constructed by P1 transduction of JC7623 with JRG2930 (aceF : : kan $\left.{ }^{\mathrm{R}}\right)$ as donor, and selecting a representative $\mathrm{Km}^{\mathrm{R}} \mathrm{Ace}^{-}$transductant (the Ace ${ }^{-}$phenotype is characterized by the inability to grow on glucose or succinate minimal media unless provided with an acetate supplement). Derivatives of JRG3141 containing the desired chromosomal replacements were recovered as $\mathrm{Ace}^{+} \mathrm{Km}^{\mathrm{s}} \mathrm{Ap}^{\mathrm{s}}$ products after transformation with pGS662-668 and designated JRG3141-3148. In the final step, the altered aceF genes were transferred from the JC7623 background to the W3110 background by selecting $\mathrm{Ace}^{+}$ $\left(\mathrm{Km}^{\mathrm{S}}\right)$ transductants of JRG2930 $\left(a c e F:: k a n^{\mathrm{R}} \mathrm{Nal}^{\mathrm{S}}\right)$ on glucose minimal medium, to provide the corresponding set of strains, JRG3162-3168.

Both sets of isogenic strains were numbered so that the multiplicity of lip segments in the chromosomal aceF gene is defined by the final digit of the strain designation. The chromosomal replacements were confirmed by PCR amplification of a diagnostic fragment containing the modified lip region using a bacterial colony as the source of template DNA and oligonucleotide primers corresponding to sequences at the $5^{\prime}$ end of the aceE gene (S361, $5^{\prime}$ GTTGATGCTTCTTATGTCGTGGTTGCGGC3'; coordinates 3635-3663, 
Stephens et al., 1983a) and within the aceF gene (S360, 3'CCACAATTGGAACGCTTTCACTTCCCGTGAC5'; coordinates 4804-4835, Stephens et al., 1983b). In each case the amplified fragment was of the size predicted for the corresponding number of lipoyl domains.

Physiological methods. The media, growth conditions and analytical methods used in quantitative physiological studies with batch and glucose-limited continuous cultures, have been described by Davé et al. (1995) and Brooke et al. (1989). The specific growth rates $\left(\mu_{\max }\right)$ were measured using cultures starting from single colonies from glucose minimal agar and developing successive overnight shake cultures of 10,50 and $100 \mathrm{ml}$ in glucose minimal medium ( $20 \mathrm{mM}$ glucose; $\mathrm{pH} 6.95$ ) to provide $100 \mathrm{ml}$ inocula for $600 \mathrm{ml}$ batch cultures in chemostat vessels operating at $\geqslant 50 \%$ air saturation and $37^{\circ} \mathrm{C}$. Growth was estimated from the optical density at $430 \mathrm{~nm}$ (Pye Unicam SP6-250 spectrophotometer) and values for $\mu_{\max }$ were calculated from the doubling times. The quoted values are means from at least three independent cultures; deviation from the mean was no more than $\pm 5 \%$. Pairwise competition experiments were performed in glucose-limited chemostat cultures $\left(20 \mathrm{mM}\right.$ glucose; $\left.D 0.075 \mathrm{~h}^{-1}\right)$ with approximately equal numbers of $\mathrm{Nal}^{\mathrm{R}}$ and $\mathrm{Nal}^{\mathrm{S}}$ bacteria in the starting population, as described by Davé et al. (1995).

\section{RESULTS}

\section{Construction of pdh operons encoding PDH complexes containing one wild-type and up to seven unlipoylatable lipoyl domains per E2p chain}

The strategy devised for constructing plasmids that encode one wild-type lipoyl domain $(+)$ and up seven mutant domains $(-)$ is summarized in Figs 1 and 2. It involved manipulating pre-existing DNA fragments with $(+-)$ and $(--+)$ configurations to produce two derivatives, $(+--)$ and $(---)$, which were then sequentially sub-cloned into the dual-replicon (pPOD6) to generate pGS641 (Fig. 1). Kanamycin-resistant resolvants of pGS641 containing two to five lip segments were then created by partial BclI digestion (or HindIII digestion in one case) followed by re-ligation, as shown in Fig. 2. Analogous plasmids containing six to eight lip segments were constructed by linearizing a derivative of the 5lip plasmid (pGS645a) with KpnI followed by partial digestion with $B c l \mathrm{I}$ and the re-ligation of different pairs of sub-fragments (Fig. 2). The KpnI-SphI fragments from each of these intermediary plasmids (pGS642-648) were transferred to pGS501 to generate a set of $P_{t a c}$ expression plasmids encoding the corresponding PDH complexes (pGS662-668). The two series of plasmids were designated $+/-$ to distinguish them from the $+/+$ series that contain multiple copies of wild-type lipoyl domains (Machado et al., 1992). The numbers of lip segments and other predicted features of all types of intermediate and ultimate plasmids were thoroughly confirmed by restriction analysis. All of the $+/$ - expression plasmids complemented the $\mathrm{Ace}^{-}$ nutritional phenotype of an aroP-pdbR-aceEF-lpdA deletion strain (JRG1342) but only in the presence of IPTG $(20 \mu \mathrm{M})$. It would thus appear that the lipoyl cofactors bound solely to the outermost domains of the E2p chains in each of $+/-$ PDH complexes confer sufficient enzymic activity to support the growth of the deletion strain.

\section{Properties of $+/-$ PDH complexes containing up to eight lipoyl domains}

After induction with IPTG, all of the expression plasmids produced sedimentable PDH complexes which could be purified by the method developed for the

Table 1. Properties of PDH complexes with multiple lipoyl domains per E2p chain

The properties of the $+/-$ series of purified PDH complexes are compared with those of the 1lipPDH complex expressed by the receptor plasmid (pGS501) and the inactive 0lip-PDH complex (pGS489). $M_{r}$ were calculated from the nucleotide sequences (without lipoic acid, Stephens $e t$ al., 1983b) or estimated from the mobilities in SDS-PAGE (mean of three gels for three preparations). The predicted $M_{r}$ for each lipoyl domain plus associated linker is approximately $\mathbf{1 1 2 5 0}$ (innermost domain) and 10000-10400 (other domains). The specific activities [units (mg protein) ${ }^{-1}$ ] are means for three preparations, the individual activities deviated $<15 \%$ from the quoted value.

\begin{tabular}{|c|c|c|c|c|c|c|c|}
\hline \multirow[t]{3}{*}{ Plasmid } & \multirow{2}{*}{\multicolumn{2}{|c|}{$\begin{array}{l}\text { Lipoyl } \\
\text { domains }\end{array}$}} & \multicolumn{3}{|c|}{$M_{\mathrm{r}}$ of E2p chain } & \multirow{3}{*}{$\begin{array}{c}\text { Increment } \\
\text { in } \boldsymbol{M}_{\mathrm{r}} \text { per } \\
\text { lipoyl } \\
\text { domain }\end{array}$} & \multirow{3}{*}{$\begin{array}{c}\text { Specific } \\
\text { activity of } \\
+/- \text { PDH } \\
\text { complex }\end{array}$} \\
\hline & & & Predicted & Apparent & Difference & & \\
\hline & Total & E2p & & & & & \\
\hline pGS489 & 0 & & 34345 & 34000 & -345 & - & $<0.001$ \\
\hline pGS501 & 1 & + & 45610 & 50600 & 4990 & 16600 & $14 \cdot 5$ \\
\hline pGS662 & 2 & $+/ 1-$ & 55592 & 65500 & 9908 & 14900 & $13 \cdot 6$ \\
\hline pGS663 & 3 & $+/ 2-$ & 65959 & 77200 & 11241 & 11700 & $11 \cdot 8$ \\
\hline pGS664 & 4 & $+/ 3-$ & 76327 & 90100 & 13773 & 20900 & $11 \cdot 9$ \\
\hline pGS665 & 5 & $+/ 4-$ & 86309 & 106000 & 19691 & 15900 & $10 \cdot 2$ \\
\hline pGS666 & 6 & $+/ 5-$ & 96677 & 116100 & 19423 & 10100 & 8.0 \\
\hline pGS667 & 7 & $+/ 6-$ & 106659 & 136800 & 30141 & 20800 & 4.9 \\
\hline pGS668 & 8 & $+/ 7-$ & 117027 & 151500 & 34473 & 14700 & $2 \cdot 1$ \\
\hline
\end{tabular}




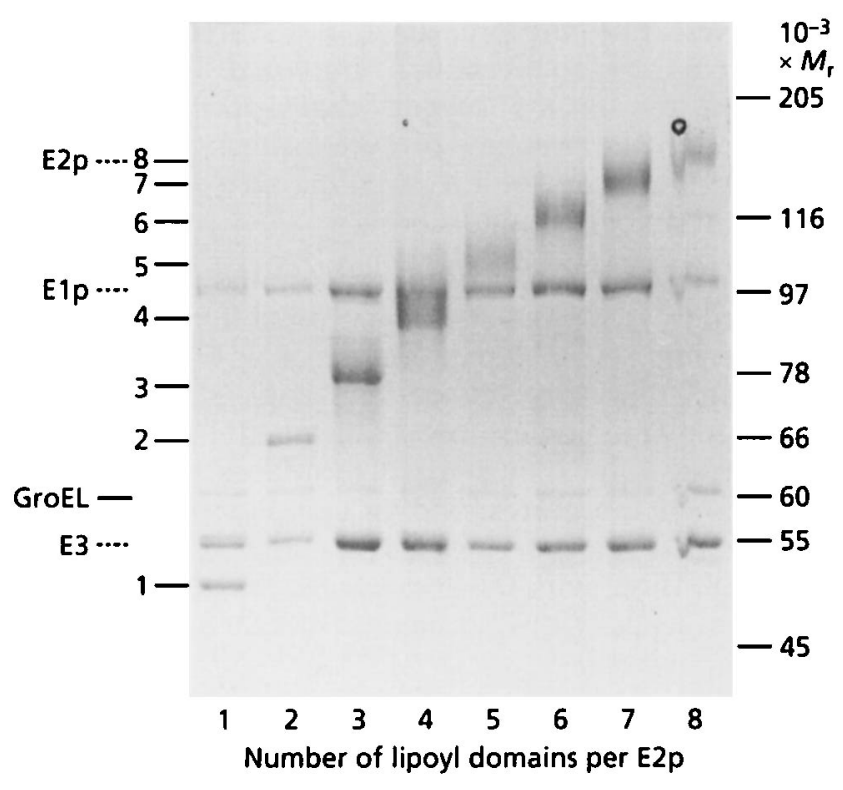

Fig. 4. SDS-PAGE analysis of purified $+/-\mathrm{PDH}$ complexes containing one to eight lipoyl domains per E2p chain. The positions of E1p, E2p (1lip-8lip), E3, and GroEL (a frequent contaminant), as well as the molecular mass markers are indicated.

amplified wild-type and $+/+$ multilip-PDH complexes (Russell et al., 1992; Machado et al., 1992). The subunit stoichiometries obtained by densitometric analysis of samples fractionated by SDS-PAGE (Fig. 4) varied within the range $0 \cdot 5-0 \cdot 7: 1 \cdot 0: 0 \cdot 9-2 \cdot 0(\mathrm{E} 1 \mathrm{p}: \mathrm{E} 2 \mathrm{p}: \mathrm{E} 3)$, and the specific activities ranged from 94 to $14 \%$ of those observed for 1lip-PDH complex expressed from the receptor plasmid (Table 1). Despite the presence of up to seven unlipoylated domains in the $+1-$ complexes, the 24 lipoyl cofactors on the outermost domains can clearly reach all three types of active site in the catalytic heart of the complex and support at least $14 \%$ of normal activity. The specific activities of the $+1-$ complexes are compared with those obtained previously for the $+/+$ series (Machado et al., 1992) in Fig. 5(a). Those of the $+/$ - series decline gradually once the number of lipoyl domains per E2p chains exceeds four, whereas those of the $+/+$ series containing $>3$ lipoyl domains per E2p chain decline in an irregular manner, but retain at least $19 \%$ of the maximum observed activity.

It had been thought that under-lipoylation might contribute to the lower activities of over-produced $+/+$ complexes, because over-expressing an independent lipoyl domain sub-gene outstrips the cell's lipoylation capacity and leads to the accumulation of unlipoylated domains (Miles \& Guest, 1987; Ali \& Guest, 1990). However, this does not apply to the over-produced 1lipPDH complex (Russell et al., 1992) nor should it apply to the $+/$ - series, where the number of lipoylatable domains (24) remains the same in all complexes. Attempts to increase the specific activities of the $+/+$ series by treating purified complexes with lipoate protein
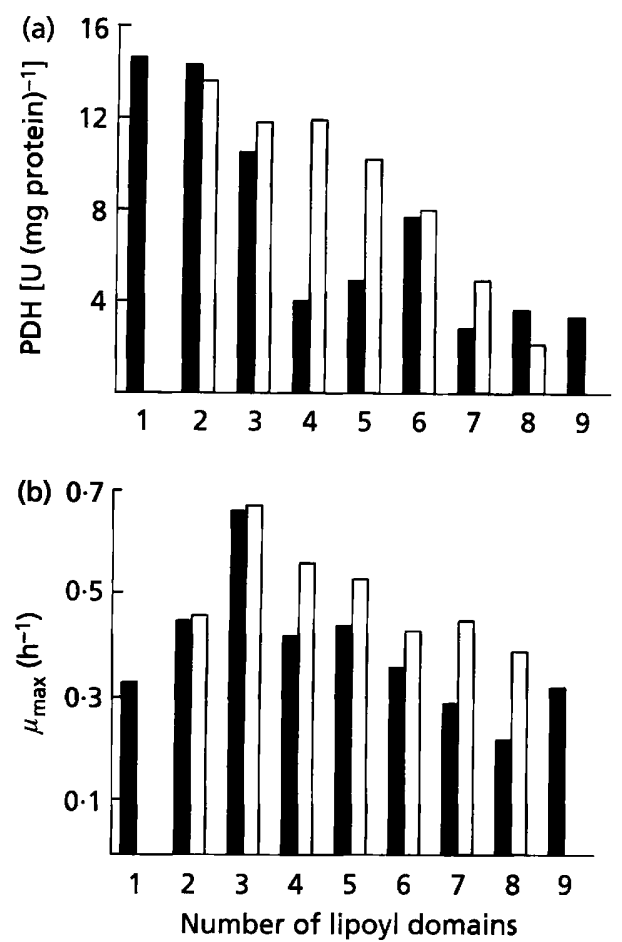

Fig. 5. Comparisons of the enzymological and physiological properties of $+/+(\square)$ and $+/-(\square)$ series of multilip-PDH complexes. (a) Specific activities of purified PDH complexes (means of three independent preparations deviating no more than $15 \%$ from the mean): $+l+$ data plotted from Machado et al. (1992) and $+/-$ data from Table 1. (b) Maximum specific growth rates $\left(\mu_{\max }\right)$ of isogenic derivatives of $W 3110$ growing on glucose minimal medium in batch culture: mean values for at least three independent cultures deviating by no more than $5 \%$ from the mean.

ligase under conditions that are known to lipoylate apodomains, were unsuccessful (data not shown). Thus it would appear that the lower specific activities of complexes containing superfluous lipoyl domains is probably due to steric interference by domains of either type $(+$ or -$)$ that are not participating in the catalytic process.

The $M_{\mathrm{r}}$ values deduced from the electrophoretic mobilities of the E2p subunits were characteristically higher than those predicted from the nucleotide sequences (Table 1). The discrepancy increases as the number of lipoyl domains increases, consistent with the view that it is due to the anomalous electrophoretic behaviour of the alanine- plus proline-rich linkers (Stephens et al., 1983b; Guest et al., 1985; Machado et al., 1992). The mean discrepancy is approximately 4000 per lipoyl domain, but the incremental changes are not uniform (Table 1). In particular, the increments associated with the addition of domains 1,4 and 7 appeared relatively high. Such non-uniformity was also observed with the $+/+$ complexes (Machado et al., 1992). The underlying reason is not known but it could be due to some form of interactive domain clustering that occurs at specific domain contents. 


\section{Construction and physiological properties of isogenic strains containing acef genes that encode multiple wild-type and mutant lipoyl domains}

Previous studies have shown that the physiological behaviour of growing bacterial cultures can serve as a very sensitive indicator of the performance of genetically modified PDH complexes (Davé et al., 1995). Indeed, such diagnostic information is obtained under conditions which avoid variations in subunit stoichiometry or differential inactivation that may occur during enzyme purification for in vitro comparisons. Two sets of isogenic derivatives of $E$. coli W3110 containing altered ace $F$ genes with multiple copies of the wild-type lip region $(+/+$ series) or one wild-type and up to seven mutant lip regions $(+/-$ series), were accordingly constructed for physiological studies. The $+/+$ strains were obtained as outlined in Fig. 3, by an allelic replacement procedure that involves the co-integration of derivatives of a thermo-sensitive replicon (pMAK705; Hamilton et al., 1989) and JRG2930 (aceF::kan $\left.{ }^{\mathrm{R}}\right)$, as used previously for lowering the lip content of the chromosomal aceF gene (Davé et al., 1995). The +/strains were obtained by transforming an aceF : $k a n^{\mathbf{R}}$ derivative of JC7623 ( $\mathrm{recBC} s b c B C$ ) with the corresponding $p d h$ expression plasmids according to Oden et al. (1990), see Methods. Both procedures involved selecting or screening for $\mathrm{Ace}^{+}$products, and made the assumption that single chromosomal copies of the altered but active $p d h$ operons would restore at least some degree of acetate-independence on glucose minimal medium. This assumption proved valid because the desired products were recovered at frequencies comparable to those observed for wild-type reconstructions.

The successful construction of both series of strains indicates that single chromosomal copies of $p d h$ operons containing one to nine wild-type lipoyl segments, or one wild-type segment with up to seven sub-terminal mutant segments, provide sufficient acetyl-CoA to support growth on plates of unsupplemented glucose minimal medium. However, the plate tests failed to reveal phenotypic differences that become apparent during batch culture in glucose minimal medium. Quantitative growth tests in liquid medium showed that the maximum specific growth rates $\left(\mu_{\max }\right)$ vary between 100 and $33 \%$ of wild-type depending on the number and type of lipoyl domains in the PDH complex (Fig. 5b). It is clear that the highest growth rates in both series are conferred by the presence of three lipoyl domains $(+++$ and $+--)$. It would also appear that extra wild-type lipoyl domains cause significantly more impairment than the same number of mutant domains (the growth rates are reproducible to within $\pm 0.5 \%$ ). However, it is not known whether this is due to a specific effect on catalysis or to an energetic burden that may be imposed by the increased demand for lipoyl cofactors. The genetically engineered strains exhibit a useful range of growth rates extending down to $33 \%$ of wild-type. However, apart from the general impairment associated with the higher orders of lipoyl domains, the growth rates are not directly related to the specific activities of the purified complexes. Furthermore, if it is assumed that the complexes are not seriously impaired during their isolation, the results suggest that specific activities measured in the presence of excess substrates, are not accurate indicators of the way the enzyme performs under physiological conditions.

Another striking observation is the similar growth rates exhibited by two pairs of strains from different sets, viz. the ++ and +-2 lip pair and the +++ and +-3lip pair (Fig. 5b). This similarity suggests that lipoylation of the outermost ( $\mathrm{N}$-terminal) lipoyl domain is of prime importance for determining the catalytic efficiency of the corresponding complex. Indeed, it is possible that the superiority of the 3lip-PDH complex relative to those with fewer domains, may depend more on the presence of three domains than the fact that they are all lipoylated.

A particularly sensitive test for the physiological superiority of one particular complex over another is provided by pairwise competition between isogenic strains growing in glucose-limited chemostat cultures. For example, when equal numbers of wild-type bacteria $(+++)$ and mutants containing two lipoyl domains per E2p chain $(++)$ are grown together, the mutant is gradually washed out, whereas in controls containing two genetically marked wild-types, both are maintained in the same proportion for over 50 generations (Davé et al., 1995). In similar chemostat competition experiments between the two 3lip strains, JRG2933 $\left(+++\mathrm{Nal}^{\mathrm{R}}\right)$ and JRG3163 (+- $\left.-\mathrm{Nal}^{\mathrm{S}}\right)$, both strains were maintained in the same relative proportions for 50 generations, deviating no more than $\pm 0.5 \%$ from the starting ratio at any stage. A more detailed comparison of the growth kinetics of W3110 and JRG3163 in glucose-limited chemostat culture $\left(D 0.082 \mathrm{~h}^{-1}\right)$ further showed that both strains exhibit the same carbon efficiencies $(52-53 \%)$, protein contents $(69-70 \%)$, biomass concentrations $\left(1.46-1.47 \mathrm{mg}\right.$ dry wt $\left.\mathrm{ml}^{-1}\right)$, carbon contents $(51 \%)$, and carbon balances $(93-95 \%)$. These observations strongly suggest that the corresponding +++ and +--3 lip-PDH complexes are functionally indistinguishable.

\section{DISCUSSION}

The strategy for creating multiple duplications of part of the aceF gene, making use of the dual replicon (pPOD6) and unique restriction sites in the duplicated region of the replaceable cassette, generates stable $p d h$ operons that express active complexes both in vivo and in vitro. The operons encoding one lipoylated domain and multiple unlipoylated domains, express high-molecularmass sedimentable complexes having subunit stoichiometries and specific activities that are not grossly different from those containing the comparable number of lipoylatable domains. The extra domains must protrude from the $\mathrm{E} 2 \mathrm{p}$ core without hindering core assembly or its ability to bind a normal complement of E1p and E3 subunits. Nevertheless, it seems remarkable that single lipoyl cofactors bound to the most peripheral 
domains of an 8lip-PDH complex can support as much as $14 \%$ of wild-type activity. The results are consistent with the view that the lipoyl cofactors approach the acetyltransferase active site from the outer surface of the core (Mattevi et al., 1992), and also with an earlier observation that a PDH complex which is totally devoid of lipoyl domains can be complemented in vivo by independent lipoyl domains expressed in trans from a genetically engineered sub-gene (Russell et al., 1989). It can also be concluded that incomplete lipoylation is not responsible for the lower catalytic activities of genetically amplified PDH complexes having $>24$ lipoyl-binding sites per complex. A major factor seems to be interference from superfluous domains that are not participating in catalysis. This probably accounts for the observed $50 \%$ reduction in catalytic activity of a $\mathrm{PDH}$ complex having a --+ configuration compared with those with having -+ and +- and ++- configurations (Allen et al., 1989).

The discrepancy between the high specific activity of the +-2 lip-PDH complex and the sub-optimal growth rate of the corresponding strain, parallels the discrepancies observed previously with the + and ++ constructs (Davé et al., 1995). Presumably the catalytic debility imposed by lowering the lipoyl domain content is subtle and detectable only under substrate-limiting conditions. Attempts to detect significant kinetic differences under such conditions are frustrated by the inability to isolate (or reassemble) purified PDH complexes of each type having reproducibly uniform overall specific activities, subunit stoichiometries and degrees of lipoylation. Indeed, the most sensitive indicator of catalytic competence would at present seem to be the specific growth rate of the corresponding isogenic strain.

The present results shed new light on the reason why the PDH complex of E. coli retains three lipoyl domains per $E 2 p$ chain. Because the inner domains need not be lipoylated in order for the 3lip-PDH complex to confer the highest growth rate in glucose minimal medium, it could be argued that domain duplication both extends the reach and improves the conformational mobility of the most peripheral lipoyl cofactors. This in turn could have the effect of facilitating optimal interactions with the three types of active sites in the assembled complex, rather than simply providing extra lipoyl cofactors that can participate in catalysis. The fact that the extra cofactors do participate in catalysis may be entirely fortuitous. The view that linker reach and quality are important is supported by the significant increase (or decrease) in specific activity that accompanies the substitution of a sub-optimal polyalanine-proline linker by a polyproline (or polyalanine) linker having the same number of residues (Turner et al., 1993). From an evolutionary standpoint, it would seem expedient to duplicate existing sequences rather than to improve the quality of the linker sequences by an incremental series of single Ala $\rightarrow$ Pro substitutions. On their own, such single substitutions would probably afford no significant selective advantage and, by increasing the $G+C$ content of the gene, might even be detrimental in the long term.
If the extra lipoyl domains are primarily fulfilling a linker function (i.e. as a spacer) there should be some reason why they are still lipoylated. Possible reasons are that they: (i) confer a selective advantage under some physiological condition not tested here; (ii) are retained by intragenic recombination with the essential wild-type lip segment; or (iii) serve as a reservoir for lipoyl cofactors. It will be interesting to study the + strain over prolonged periods to see whether any ,+-+++- , or +++ revertants/recombinants arise, and to set up pairwise competition experiments to find conditions in which the possession of a lipoylatable $(+)$ rather than an unlipoylatable (-) spacer might confer a selective advantage.

The 16 isogenic strains containing wild-type or genetically engineered $p d h$ operons now offer for specific purposes a choice of strains whose growth rates and carbon fluxes through the PDH complex are endogenously pre-set over a wide range (33-100\% of wild-type). The strains were stable in the cultures studied here but a major factor affecting their usefulness might be their long-term stability. It will in the future be interesting to examine the effects of prolonged cultivation under glucose-limiting conditions with strains containing $<3$ or $>3$ lipoyl domains per E2p chain, to see whether 3lip 'revertants' are selected by internal gene duplications or deletions. The present set of engineered strains, and the strain containing an exogenously regulated $P_{t a c}-a c e E F-$ lpdA operon, provide opportunities for redirecting metabolic carbon to commercially important products and for off-setting the deleterious accumulation of acetate.

\section{ACKNOWLEDGEMENTS}

We are indebted to A. F. C. Perry and N. L. Brown for providing the double replicon, and to $R$. E. Roberts and $M$. Luttik for technical assistance. The work was supported by a project grant from the Biotechnology Directorate of the BBSRC (J.R.G. and M.M.A.) and research studentships from the CNP $_{q}$ Brazil (R.S.M.) and The Kuwait Institute for Scientific Research (K.Y.M.).

\section{REFERENCES}

Ali, S. T. \& Guest, J. R. (1990). Isolation and characterization of lipoylated and unlipoylated domains of the E2p subunit of the pyruvate dehydrogenase complex of Escherichia coli. Biochem J 271, 139-145.

Allen, A. G., Perham, R. N., Allison, N., Miles, J. S. \& Guest, J. R. (1989). Reductive acetylation of tandemly repeated lipoyl domains in the pyruvate dehydrogenase multienzyme complex of Escherichia coli is random order. $J$ Mol Biol 208, 623-633.

Brooke, A. G., Watling, E. M., Attwood, M. M. \& Tempest, D. W. (1989). Environmental control of metabolic fluxes in thermotolerant methylotrophic Bacillus strains. Arch Microbiol 151, 268-273.

Brookfield, D. E., Green, J., Ali, S. T., Machado, R. S. \& Guest J. R. (1991). Evidence for two protein-lipoylation activities in Escherichia coli. FEBS Lett 295, 13-16.

Cassey, B., Attwood, M. M. \& Guest, J. R. (1996). Environmental control of pyruvate dehydrogenase complex expression in 
Escherichia coli. In Microbial Physiology and Gene Regulation: Emerging Principles and Applications, pp. 315-316. Beijerinck Centennial Symposium, The Hague, Netherlands (December 1995).

Chang, Y.-Y., Wang, A.-Y. \& Cronan, J. E. (1994). Expression of Escherichia coli pyruvate oxidase (PoxB) depends on the sigmafactor encoded by the $r p o S(k a t F)$ gene. Mol Microbiol 11, 1019-1028.

Cutayer, J. M. \& Poillon, D. (1989). High cell density culture of $E$. coli in a fed-batch system with dissolved oxygen as substrate feed indicator. Biotechnol Lett 11, 155-160.

Davé, E., Guest, J. R. \& Attwood, M. M. (1995). Metabolic engineering in Escherichia coli: lowering the lipoyl domain content of the PDH complex adversely affects the growth rate and yield. Microbiology 141, 1839-1849.

Green, D. E., Morris, T. W., Green, J., Cronan, J. E. \& Guest, J. R. (1995). Purification and properties of the lipoate protein ligase of Escherichia coli. Biochem J 309, 853-862.

Guest, J. R., Angier, S. J. \& Russell, G. C. (1989). Structure, expression, and protein engineering of the pyruvate dehydrogenase complex of Escherichia coli. Ann NY Acad Sci 573, 76-99.

Guest, J. R., Lewis, H. M., Graham, L. D., Packman, L. C. \& Perham, R. N. (1985). Genetic reconstruction and functional analysis of the repeating lipoyl domains in the pyruvate dehydrogenase multienzyme complex of Escherichia coli. J Mol Biol 185, 743-754.

Guest, J. R., Quail, M. A., Davé, E., Cassey, B. \& Attwood, M. M. (1996). Regulatory and other aspects of pyruvate dehydrogenase complex synthesis in Escherichia coli. In Biochemistry and Physiology of Thiamin Diphosphate Enzymes, pp. 326-333. Edited by $\mathrm{H}$. Biswanger \& A. Schellenberger. Prien: A. \& C. Intemann.

Hamilton, C. M., Aldea, M., Washburn, B. K., Babitzke, P. \& Kushner, S. R. (1989). New method for generating deletions and gene replacements in Escherichia coli. J Bacteriol 171, 4617-4622.

Kaiser, M. \& Sawers, G. (1994). Pyruvate formate-lyase is not essential for nitrate respiration by Escherichia coli. FEMS Microbiol Lett 117, 163-168.

Konstantinov, K., Kishimoto, M., Seki, T. \& Yoshida, T. (1990). A balanced DO-stat and its application to the control of acetic acid excretion by recombinant Escherichia coli. Biotechnol Bioeng 36, 750-758.

Machado, R. S., Clark, D. P. \& Guest, J. R. (1992). Construction and properties of pyruvate dehydrogenase complexes with up to nine lipoyl domains per lipoate acetyltransferase chain. FEMS Microbiol Lett 100, 243-248.

Machado, R. S., Guest, J. R. \& Williamson, M. P. (1993). Mobility in pyruvate dehydrogenase complexes with multiple lipoyl domains. FEBS Lett 323, 243-246.

Mattevi, A., de Kok, A. \& Perham, R. N. (1992). The pyruvate dehydrogenase multienzyme complex. Curr Opin Struct Biol 2 , 877-887.

Miles, J. S. \& Guest, J. R. (1987). Subgenes expressing single lipoyl domains of the pyruvate dehydrogenase complex of Escherichia coli. Biochem J 245, 869-874.

Miles, J. S., Guest, J. R., Radford, S. E. \& Perham, R. N. (1988). Investigation of the mechanism of active site coupling in the pyruvate dehydrogenase multienzyme complex of Escherichia coli by protein engineering. $J \mathrm{Mol} B i o l$ 202, 97-106.

Miller, J. H. (1972). Experiments in Molecular Genetics. Cold Spring Harbor, NY: Cold Spring Harbor Laboratory.

Morris, T. W., Reed, K. E. \& Cronan, J. E. (1995). Lipoic acid metabolism in Escherichia coli. The $l p l A$ and lipB genes define redundant pathways for ligation of lipoyl groups to apoprotein. J Bacteriol 177, 1-10.

Oden, K. L., DeVeaux, L. C., Vibat, C. R. T., Cronan, J. E. \& Gennis, R. B. (1990). Genomic replacement in Escherichia coli K-12 using covalently closed circular plasmid DNA. Gene 96, 29-36.

Perham, R. N. (1991). Domains, motifs and linkers in 2-oxo acid dehydrogenase multienzyme complexes: a paradigm in the design of a multifunctional protein. Biochemistry 30, 8501-8512.

Russell, G. C. \& Guest, J. R. (1990). Overexpression of restructured pyruvate dehydrogenase complexes and site-directed mutagenesis of a potential active-site histidine residue. Biochem J 269, 443-450.

Russell, G. C., Williamson, R. A. \& Guest, J. R. (1989). Partial complementation of pyruvate dehydrogenase deficiency by independently expressed lipoyl and catalytic domains of the dihydrolipoamide acetyltransferase component. FEMS Microbiol Lett 60, 267-272.

Russell, G. C., Machado, R. S. \& Guest, J. R. (1992). Overproduction of the pyruvate dehydrogenase multienzyme complex of Escherichia coli and site-directed substitutions in the E1p and E2p subunits. Biochem J 287, 611-619.

Sambrook, J., Fritsch, E. F. \& Maniatis, T. (1989). Molecular Cloning: a Laboratory Manual, 2nd edn. Cold Spring Harbor, NY: Cold Spring Harbor Laboratory.

Schulze, E., Westphal, A. H., Veenhuis, M. \& de Kok, A. (1992). Purification and cellular localization of wild type and mutated dihydrolipoyltransacetylases from Azotobacter vinelandii and Escherichia coli expressed in E. coli. Biochim Biophys Acta 1120, 87-96.

Stephens, P. E., Darlison, M. G., Lewis, H. M. \& Guest, J. R. (1983a). The pyruvate dehydrogenase complex of Escherichia coli K12. Nucleotide sequence encoding the pyruvate dehydrogenase component. Eur J Biochem 133, 155-162.

Stephens, P. E., Darlison, M. G., Lewis, H. M. \& Guest, J. R. (1983b). The pyruvate dehydrogenase complex of Escherichia coli K12. Nucleotide sequence encoding the dihydrolipoamide acetyltransferase component. Eur J Biochem 133, 481-489.

Turner, S. L., Russell, G. C., Williamson, M. P. \& Guest, J. R. (1993). Restructuring an interdomain linker in the dihydrolipoamide acetyltransferase component of the pyruvate dehydrogenase complex of Escherichia coli. Protein Eng 6, 101-108.

Received 9 August 1996; revised 24 September 1996; accepted 26 September 1996. 